Research Article

\title{
The Effect of Charcot Neuroarthropathy on Limb Preservation in Diabetic Patients with Foot Wound and Critical Limb Ischemia after Balloon Angioplasty
}

\author{
Mehmet Burak Çildağ and Ömer Faruk Kutsi Köseoğlu \\ Department of Interventional Radiology, Adnan Menderes University Medicine Faculty, 09100 Aydın, Turkey \\ Correspondence should be addressed to Mehmet Burak Çildağ; mbcildag@yahoo.com
}

Received 12 May 2017; Revised 17 July 2017; Accepted 1 August 2017; Published 29 August 2017

Academic Editor: Andrea Flex

Copyright @ 2017 Mehmet Burak Çildağ and Ömer Faruk Kutsi Köseoğlu. This is an open access article distributed under the Creative Commons Attribution License, which permits unrestricted use, distribution, and reproduction in any medium, provided the original work is properly cited.

\begin{abstract}
Objective. The aim of this article is to investigate one-year limb preservation rates after below-the-knee angioplasty in patients with diabetic foot wound who only have critical limb ischemia (CLI) and those who have Charchot neuroarthropathy (CN) accompanied by CLI. Methods. This single-center, retrospective study consists of 63 patients with diabetic foot wound who had undergone lower extremity balloon angioplasty of at least 1 below-the-knee (BTK) vessel. Only those patients with postprocedural technical success of $100 \%$ were selected from the database. All patients were classified into two groups as patients with CLI and CN and patients with CLI only without CN. The Kaplan-Meier method was used to compare the limb preservation rates for the two groups. Results. There was no statistically significant difference between patient age, gender, diabetic disease duration, and comorbid disease such as chronic renal insufficiency, hypertension, and coronary artery disease of the two groups $(p>0.05)$. Limb preservation in the 12 months was $59.1 \%$ in the $\mathrm{CN}$ group and $92.7 \%$ in the group without $\mathrm{CN}$. Also, limb preservation rates between the two groups displayed statistically significant differences $(p<0.005)$. Conclusion. This study showed that CLI can accompany CN in patients with diabetes. Limb preservation rates with endovascular treatment in diabetic patients with CLI only are better than in diabetic patients with CLI and CN.
\end{abstract}

\section{Introduction}

Peripheral artery disease (PAD) can be defined as a group of disorders characterized by narrowing and obstruction of the arteries that reduce blood flow. Critical limb ischemia (CLI) is a clinical condition characterized by PAD-related ischemic tissue loss including incurable ulcers or gangrene and ischemic pain at rest. Within 1 year of CLI diagnosis, $30 \%$ of cases undergo major amputation and 25-30\% die [1]. Patients who develop CLI require early revascularization due to poor prognosis. CLI is common in diabetic patients and belowthe-knee (BTK) arteries are usually affected [2]. In recent years, endovascular therapy has replaced surgical bypass treatment as a revascularization method especially in BTK arteries. Endovascular revascularization is now the first choice in CLI cases of BTK level $[3,4]$. Also, Charcot neuroarthropathy $(\mathrm{CN})$ is the other causative condition of limb loss in patients with diabetes. $\mathrm{CN}$ is a progressive disease involving the foot and ankle bones, joints, and soft tissues. It occurs in $0.1-7.5 \%$ of all patients with diabetes and $29 \%$ of diabetes patients with peripheral neuropathy $[5,6]$. Major amputation rate increases especially in $\mathrm{CN}$ accompanied by ulcers or osteomyelitis [7]. Apart from a few studies, the literature has insufficient studies reporting PAD or CLI incidence in $\mathrm{CN}$ [8]. Due to the natural course of diabetes mellitus, $\mathrm{PAD}$ and CLI can be expected in CN cases. According to our clinical observations, the coexistence of CN and CLI is not so rare. We sometimes even have difficulty in differentiating whether it is due to ischemic or neuropathic origin when a diabetic patient with $\mathrm{CN}$ develops a foot wound and consider the case as neuroischemic. In addition, in cases with foot wound and CN accompanied by CLI, delayed wound healing after minor amputation and secondary infection may occur which ultimately may require major 
amputation. Although there are studies in the literature showing the effectiveness of endovascular treatment in cases with diabetic foot wound and CLI, there are no studies showing the effectiveness of endovascular treatment in cases with $\mathrm{CN}$ accompanied by CLI.

In this study, we investigated one-year limb preservation rates after angioplasty in patients with diabetic foot wound who only have CLI, and those who have CN accompanied by CLI.

\section{Material and Method}

2.1. Study Population and Design. This is a retrospective, single-center study based on the collected data of patients with diabetic wound who had undergone lower extremity percutaneous balloon angioplasty (PBA) of at least 1 BTK vessel. After institutional review board approval, imaging data between October 2014 and March 2016 were reviewed.

Inclusion criteria were the presence of diabetic foot wound and CLI, patients who had plain radiography of a damaged foot and patients who had undergone balloon angioplasty for stenosis or occlusion of at least 1 BTK vessel with distal runoff to the foot with technical success.

Exclusion criteria were planned major amputation before angiography and unsuccessful PTA, patients whose data constituted any intervention before angioplasty, who did not have plain radiography of the foot, who had suspicion of acute $\mathrm{CN}$, and who had concomitant above-knee arterial steno-occlusive lesions including the aortoiliac and femoropopliteal arterial lesions.

$\mathrm{CN}$ diagnosis was made based on clinical observations such as foot deformities and direct graphy findings such as subluxation or dislocation and erosion or destruction of foot and ankle joints. Screening was done by magnetic resonance angiography before angioplasty in all patients to determine the affected BTK arteries. Interventions had been performed by antegrade approach and with the use of $5 \mathrm{~F}$ sheaths. The ratio of balloon to vessel diameter had been planned to be $1: 1$. The balloons available during the study period had a diameter of 2.5 to $3.0 \mathrm{~mm}$ and a length of 60 to $120 \mathrm{~mm}$. In case of flow-limiting dissection or residual stenosis of $>30 \%$, a prolonged dilation had been performed. Postintervention dual antiplatelet therapy with $100 \mathrm{mg}$ aspirin and $75 \mathrm{mg}$ clopidogrel once daily had been given for at least one month, and $100 \mathrm{mg}$ aspirin had been given daily thereafter. Technical success was defined as restoration of direct flow in the target vessel with runoff to the foot and a residual stenosis of $<30 \%$. Once discharged, patients were followed-up in a multidisciplinary, dedicated foot clinic to facilitate the healing process and recovery of ambulatory function.

We chose 1 year prevention of amputation as the end point of this study, and amputation was defined as limb loss below or above the knee. All angiographic images and plain radiographies transferred from the radiology database were evaluated in the workstation by a radiologist with 14 years of experience. All patients were classified into two groups as diabetic patients with CLI and CN and diabetic patients with CLI only without CN.
2.2. Statistical Analysis. Statistical analyses were performed using Statistical Package for the Social Sciences (SPSS) 17.0 statistical software for Windows (SPSS Inc., Chicago, IL, USA). Comparisons between patients with and without Charcot neuroarthropathy were performed using the $t$-test for continuous variables and the $\chi 2$ test for discrete variables. Kaplan-Meier life table analysis was used to calculate limb preservation of the two groups. The log-rank test was used to compare the limb preservation rates of the groups with $\mathrm{CN}$ and without $\mathrm{CN}$ and to determine statistically significant levels. $p$ values $<0.05$ were considered statistically significant.

\section{Results}

63 patients met inclusion and exclusion criteria during the study period. Thus, the study consists of 63 patients with diabetic foot. Of these patients, $44(69.8 \%)$ were men and $19(30.2 \%)$ were women with a mean age of 67.2 years (ranging 51-84). The mean disease (diabetes mellitus) duration of all patients was 22.2 years (ranging $8-32$ ). The most common comorbid disease was chronic renal insufficiency (34.9\%). Baseline clinical characteristics were similar between the study groups. Treated lesions had a high degree of complexity in both study arms; $84.1 \%$ of the lesions were total occlusions. None of the patients in either study arms underwent inflow lesion treatment. In 12 months, the overall limb preservation rate was $81 \%(51 / 63)$, and the mean survival time of limb preservation after PBA was 11.07 months ( $\operatorname{std} \pm 0.26$ ).

3.1. Diabetic Patients with CLI and CN Group. 22 (34.9\%) patients with diabetic foot with $\mathrm{CN}$ underwent conventional angiography. CN was in the right foot in $11 / 22$; left foot, 10/22; and bilateral, $1 / 22$. In 12 months, limb preservation was $59.1 \%$ $(13 / 22)$. The mean survival time of limb salvage after PBA was 9.95 months ( $\mathrm{std} \pm 0.57)$.

3.2. Diabetic Patients with CLI and without CN Group. 41 (65.1\%) patients with diabetic foot without CN underwent conventional angiography. In 12 months, limb preservation was $92.7 \%$ (38/41). The mean survival time of limb salvage after PBA was 11.68 months (std \pm 0.20 ).

There was no statistically significant difference between patient age, gender, diabetic disease duration, and comorbid disease such as chronic renal insufficiency, hypertension, and coronary artery disease of the two groups $(p>0.05)$. Demographic characteristics of the patients are shown in Table 1. Limb preservation rates between $\mathrm{CN}$ and without $\mathrm{CN}$ group displayed statistically significant differences in the 12 months (Figure 1). Also, there was a statistically significant difference between the two groups in mean limb salvage time $(p<0.005)$.

\section{Discussion}

$\mathrm{CN}$ is a condition associated with peripheral neuropathy common in diabetic patients, characterized by joint and bone fractures, dislocation, and foot deformities [9]. It can be diagnosed clinically and radiologically, and its treatment is primarily conservative. The aim of the treatment is to create a plantigrade foot which provides bone stability $[10,11]$. A 
TABLE 1: Demographic characteristics and occlusive artery numbers of diabetic patients with Charcot neuroarthropathy and without Charcot neuroarthropathy.

\begin{tabular}{|c|c|c|c|c|}
\hline & $\begin{array}{l}\text { Overall patients } \\
n: 63\end{array}$ & $\begin{array}{l}\text { Patients with CLI and CN } \\
n: 22\end{array}$ & $\begin{array}{l}\text { Patients with CLI and without CN } \\
n: 41\end{array}$ & \\
\hline Gender (\% male) & $44(69.8 \%)$ & $14(66 \%)$ & $25(61 \%)$ & $p: 0.67$ \\
\hline Age (years) & $67.21(\operatorname{std} \pm 8.30)$ & $66.59(\operatorname{std} \pm 8.87)$ & $67.54(\mathrm{std} \pm 8.07)$ & $p: 0.59$ \\
\hline DM disease duration (years) & $22.2(\operatorname{std} \pm 12.6)$ & $23.4(\operatorname{std} \pm 11.6)$ & $21.2(\operatorname{std} \pm 13.1)$ & $p: 0.66$ \\
\hline \multicolumn{5}{|l|}{ Comorbid ( $n: 54)$} \\
\hline Chronic renal insufficiency & $24(38 \%)$ & $8(36.3 \%)$ & $16(39 \%)$ & \\
\hline Coronary artery disease & $10(15.9 \%)$ & $4(18.1 \%)$ & $6(14.6 \%)$ & $p: 0.73$ \\
\hline Hypertension & $10(15.9 \%)$ & $3(13.6 \%)$ & $7(17.1 \%)$ & \\
\hline Patients with occlusion and stenosis & 53 & $19(86.3 \%)$ & $34(82.9 \%)$ & $p: 0.65$ \\
\hline Patients with stenosis only & 10 & $3(13.6 \%)$ & $7(17.1 \%)$ & $p: 0.89$ \\
\hline
\end{tabular}

n: number; CN: Charcot neuroarthropathy; std: standard deviation; DM: diabetes mellitus.

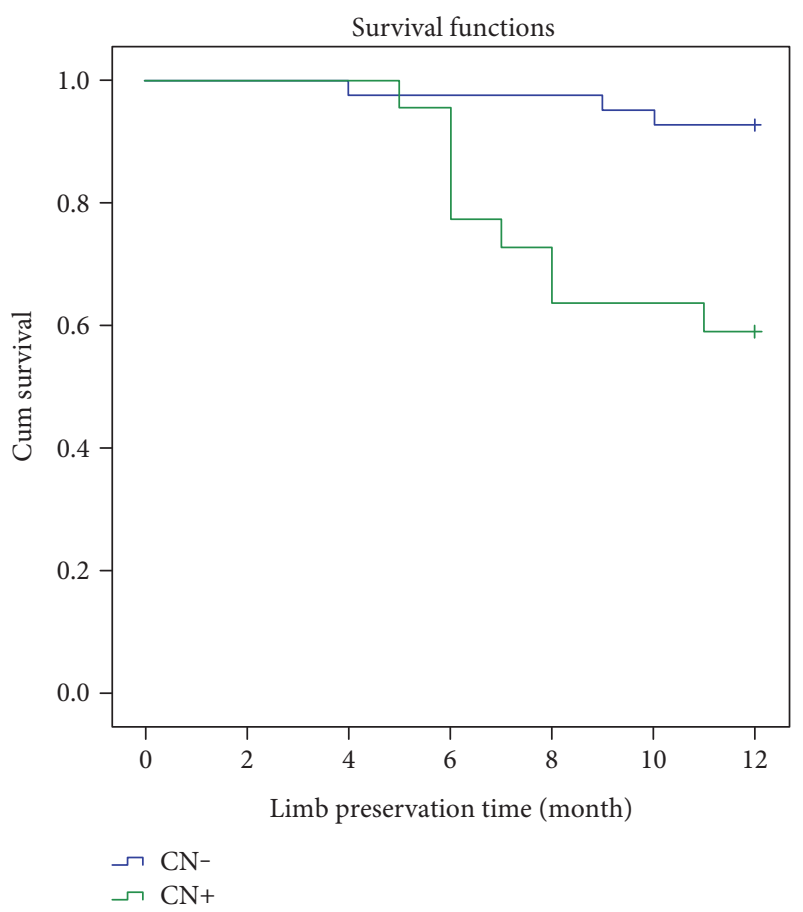

FIGURE 1: Kaplan-Meier graph comparing the limb preservation rates after endovascular treatment in diabetic patients with only CLI and in diabetic patients CLI with CN.

stable plantigrade foot may reduce the development of foot ulcers. Developing ulcers in CN increase the risk of major amputation, and patients with diabetes and Charcot deformity associated with $\mathrm{PAD}$ also have a major risk of ulceration and infection [7]. CLI can be defined as a serious form of PAD that describes patients with chronic ischemic rest pain or with ischemic skin lesions, either ulcers or gangrene. Although amputation rates are not clearly known in patients with $\mathrm{CN}$, patients with $\mathrm{CN}$ and accompanying foot ulcers have been shown to be 12 times more likely to have amputation risk than patients with CN alone [12]. Treatment of CLI in patients with $\mathrm{CN}$ is important in patients with impaired ulcer healing because patients with $\mathrm{CN}$ have a greater risk of infectious complications after surgery. Although relative ischaemia is a common contributing factor in complications of the foot in diabetes, there was not sufficient information between $\mathrm{CN}$ and CLI. Arterial pathology is most commonly shown to involve BTK arteries in patients with diabetes [13-16]. Revascularization in BTK arteries can be performed by surgical or endovascular methods. In recent years, endovascular revascularization, which is more comfortable, has been performed as the first choice treatment in elderly diabetic patients with lower morbidity and mortality rates, who do not require general anesthesia, especially with a high likelihood of having comorbid diseases [3, 4]. Lida et al. [17] identified diabetes as one of the factors associated with major amputation after endovascular therapy for patients with CLI due to isolated below-the-knee lesions, and they found limb preservation rate at 2 years to be $68 \%$ in patients with diabetes. Another study by Ferraresi et al. [18] found a limb preservation rate of $93 \%$ at a mean follow-up of 1048 days after infrapopliteal angioplasty in diabetic patients with CLI. Ryu et al. [19] compared clinical outcome after infrapopliteal angioplasty in CLI patients with and without diabetes and reported that the primary patency rate is lower in patients with diabetes although there was no significant difference in the limb preservation rate. Recently, Tartaglia et al. [20] found a one-year limb preservation rate of $84 \%$ after infrapopliteal angioplasty in patients at high risk of diabetes. All these studies showed the effectiveness of angioplasty on limb preservation in patients with diabetes and CLI, but there was no published study about limb preservation after angioplasty in patients with $\mathrm{CN}$ accompanied by CLI. In our study, the one-year limb preservation rate in all patients with ischemic diabetic foot wound who underwent endovascular treatment of BTK arteries was found to be $81 \%$. This rate was found to be $92.7 \%$ in patients without $\mathrm{CN}$ and $59.1 \%$ in cases with $\mathrm{CN}$. Limb preservation rates in cases without $\mathrm{CN}$ are similar to those in the studies in the literature. However, the lower rates in cases with $\mathrm{CN}$ are considered to be due to the accompanying ischemic neuropathy in these cases. 
Several limitations of the present study need to be considered. Firstly, this was a retrospective study from a single institution with a small number of patients. Secondly, we did not perform follow-up angiography or use other imaging modalities for patency of the treated vascular bed. Thirdly, it is unclear whether the cause of amputation in major amputation cases was ischemia or neuropathy.

In conclusion, this study showed that diabetic $\mathrm{CN}$ patients may be accompanied by CLI and neuropathies may accompany ischemia in nonhealing foot ulcers. Although endovascular treatment has been shown to have higher limb preservation rates in patients who only have CLI without $\mathrm{CN}$, major amputation rate is considered to be reduced with evaluation of BTK arteries and additional endovascular therapy in diabetic patients with $\mathrm{CN}$. Even if surgery is planned for $\mathrm{CN}$, according to us, $\mathrm{PBA}$ treatment before surgery is useful for limb preservation in diabetic patients with CN accompanied by CLI. However, there is a need for studies with a larger number of patients with $\mathrm{CN}$.

\section{Conflicts of Interest}

The authors have no conflict of interest.

\section{References}

[1] A. J. Feiring, M. Krahn, L. Nelson, A. Wesolowski, D. Eastwood, and A. Szabo, "Preventing leg amputations in critical limb ischemia with below-the-knee drug eluting stents: the PARADISE (preventing amputations using drug eluting stents) trial," Journal of the American College of Cardiology, vol. 55, pp. 1580-1589, 2010.

[2] D. Clair, S. Shah, and J. Weber, "Current state of diagnosis and management of critical limb ischemia," Current Cardiology Reports, vol. 14, pp. 160-170, 2012.

[3] L. Norgren, W. R. Hiatt, J. A. Dormandy et al., "Inter-society consensus for the management of peripheral arterial disease," International Angiology, vol. 26, pp. 81-157, 2007.

[4] H. van Overhagen, S. Spiliopoulos, and D. Tsetis, "Below-theknee interventions," Cardiovascular and Interventional Radiology, vol. 36, pp. 302-311, 2013.

[5] K. A. Chisholm and J. M. Gilchrist, "The Charcot joint: a modern neurologic perspective," Journal of Clinical Neuromuscular Disease, vol. 13, pp. 1-13, 2011.

[6] S. M. Rajbhandari, R. C. Jenkins, C. Davies, and S. Tesfaye, "Charcot neuroarthropathy in diabetes mellitus," Diabetologia, vol. 45, pp. 1085-1096, 2002.

[7] C. Caravaggi, A. B. Sgarzaroli, P. Galenda et al., "Long-term follow-up of tibiocalcaneal arthrodesis in diabetic patients with early chronic Charcot osteoarthropathy," The Journal of Foot and Ankle Surgery, vol. 51, pp. 408-411, 2012.

[8] D. K. Wukich, K. M. Raspovic, and N. C. Suder, "Prevalence of peripheral arterial disease in patients with diabetic Charcot neuroarthropathy," The Journal of Foot and Ankle Surgery, vol. 55, pp. 727-731, 2016.

[9] D. L. Nielson and D. G. Armstrong, "The natural history of Charcot's neuroarthropathy," Clinics in Podiatric Medicine and Surgery, vol. 25, pp. 53-62, 2008.
[10] D. K. Wukich and W. Sung, "Charcot arthropathy of the foot and ankle: modern concepts and management review," Journal of Diabetes and its Complications, vol. 23, pp. 409-426, 2009.

[11] P. R. Burns and D. K. Wukich, "Surgical reconstruction of the Charcot rearfoot and ankle," Clinics in Podiatric Medicine and Surgery, vol. 25, pp. 95-120, 2008.

[12] M. W. Sohn, R. M. Stuck, M. Pinzur, T. A. Lee, and E. B. Mak, "Lower-extremity amputation risk after Charcot arthropathy and diabetic foot ulcer," Diabetes Care, vol. 33, pp. 98$100,2010$.

[13] M. D. McDaniel and J. L. Cronenwett, "Basic data related to the natural history of intermittent claudication," Annals of Vascular Surgery, vol. 3, pp. 273-277, 1989.

[14] R. Ferraresi, L. M. Palena, G. Mauri, and M. Manzi, "Interventional treatment of the below the ankle peripheral artery disease," in PanVascular Medicine, P. Lanzer, Ed., Springer-Verlag, New York, 2nd edition, 2015.

[15] J. A. Dormandy and G. D. Murray, "The fate of the claudicant-a prospective study of 1969 claudicants," European Journal of Vascular Surgery, vol. 5, pp. 131-133, 1991.

[16] R. S. Most and P. Sinnock, "The epidemiology of lower extremity amputations in diabetic individuals," Diabetes Care, vol. 6, pp. 87-91, 1983.

[17] O. Lida, Y. Soga, K. Hirano et al., "Midterm outcomes and risk stratification after endovascular therapy for patients with critical limb ischemia due to isolated below-the-knee lesions," European Journal of Vascular and Endovascular Surgery, vol. 43, pp. 313-321, 2012.

[18] R. Ferraresi, M. Centola, M. Ferlini et al., "Long-term outcomes after angioplasty of isolated, below-the-knee arteries in diabetic patients with critical limb ischaemia," European Journal of Vascular and Endovascular Surgery, vol. 37, pp. 336-342, 2009.

[19] H. M. Ryu, J. S. Kim, Y. G. Ko, M. K. Hong, Y. Jang, and D. H. Choi, "Comparison of clinical outcome of infrapopliteal angioplasty between Korean diabetic and non-diabetic patients with critical limb ischemia," Circulation Journal, vol. 76, pp. 335-341, 2012.

[20] E. Tartaglia, A. Lejay, Y. Georg, M. Roussin, F. Thaveau, and N. Chakfe, "Results of isolated infrapopliteal percutaneous transluminal angioplasty for critical limb ischemia in highrisk diabetic patients," Vascular, vol. 24, pp. 515-522, 2016. 


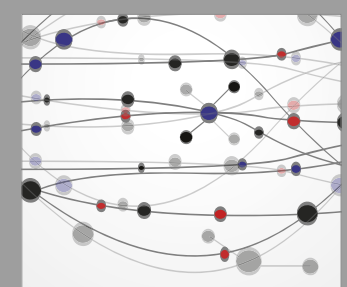

The Scientific World Journal
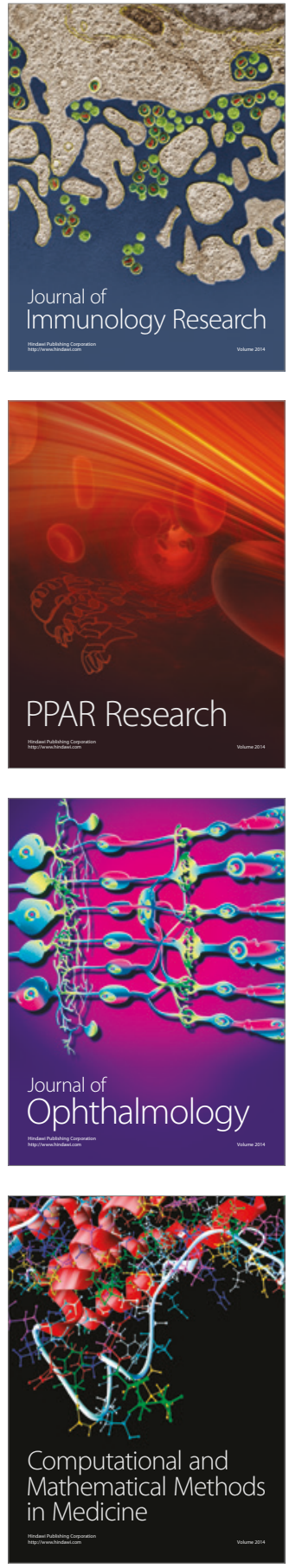

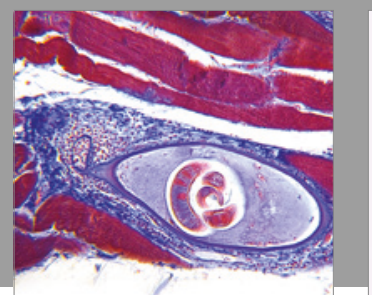

Gastroenterology Research and Practice
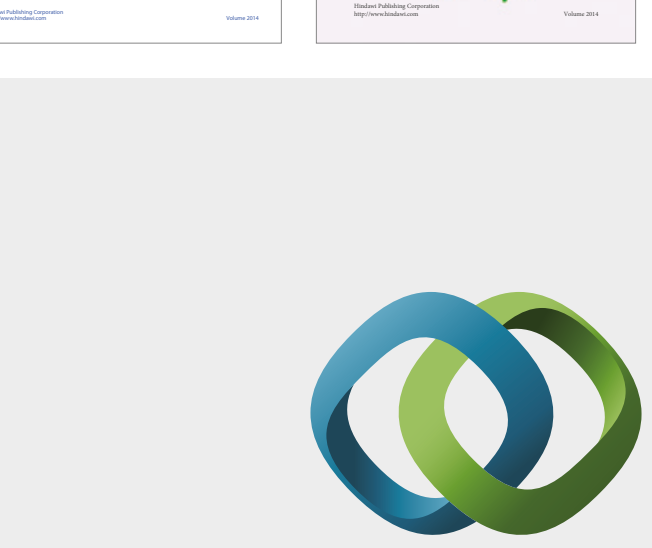

\section{Hindawi}

Submit your manuscripts at

https://www.hindawi.com
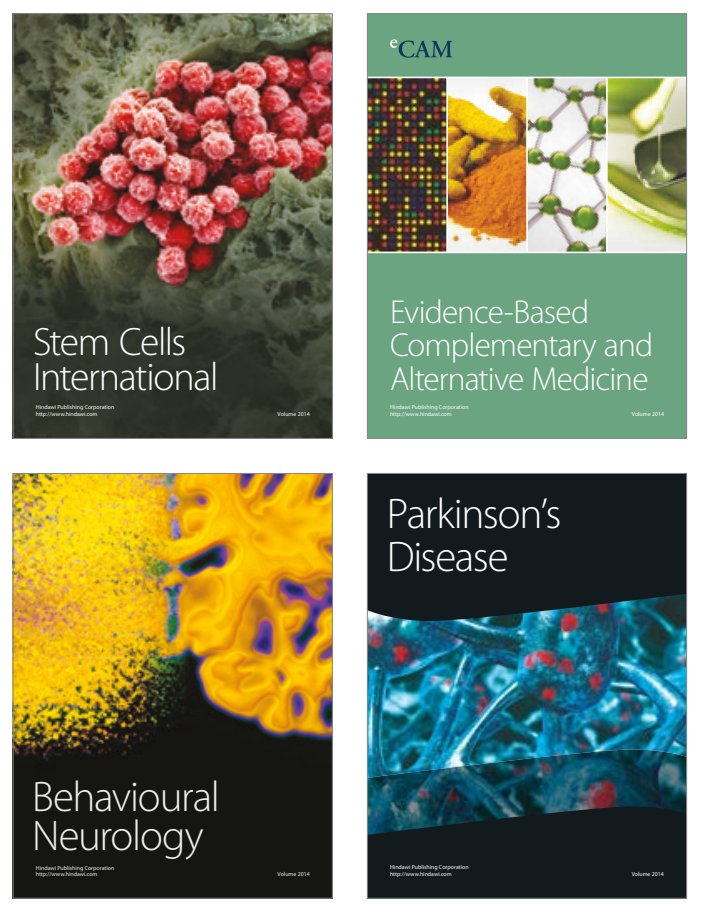
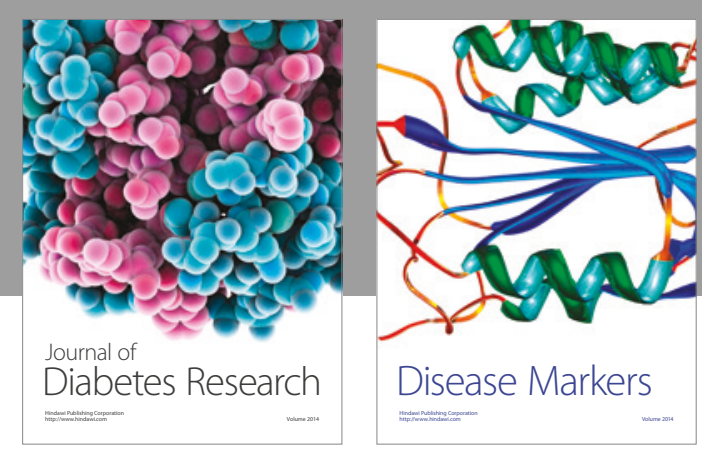

Disease Markers
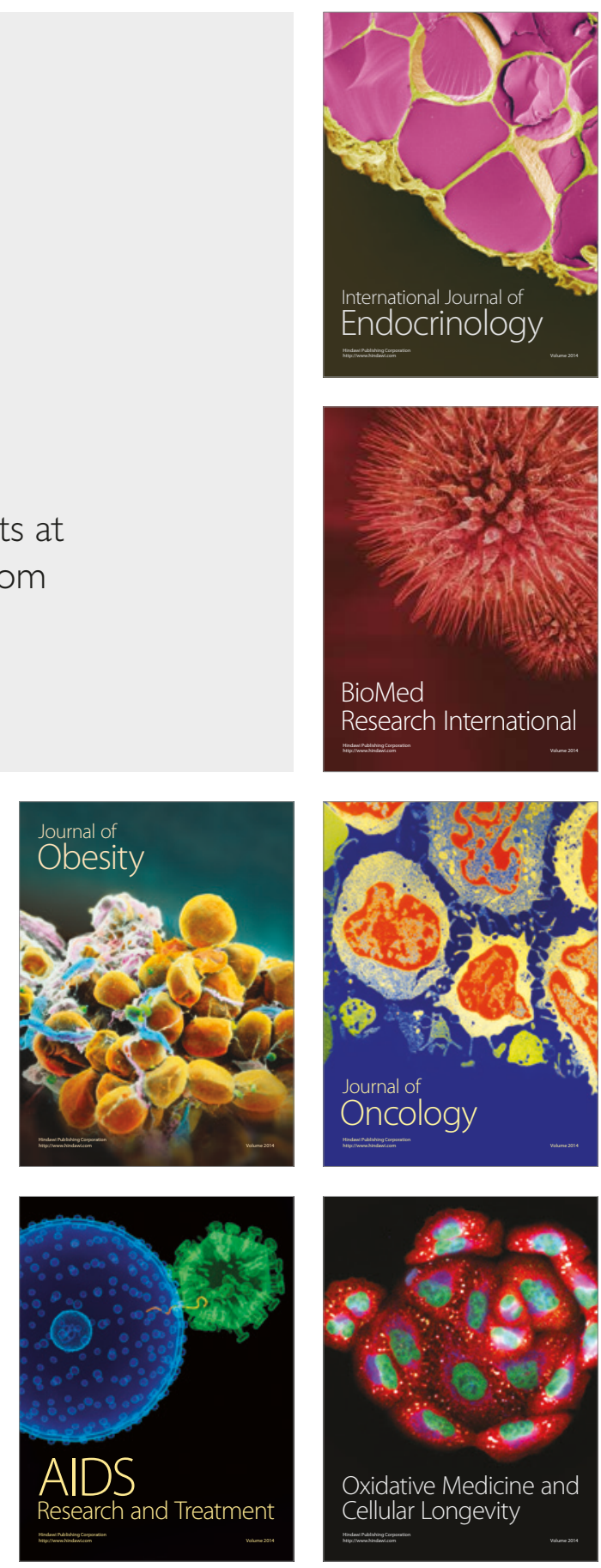